\title{
Hydrolysed formula and risk of allergic or autoimmune disease: systematic review and meta-analysis
}

\author{
Robert J Boyle,1 Despo lerodiakonou, ${ }^{1,2}$ Tasnia Khan, ${ }^{1}$ Jennifer Chivinge, ${ }^{1}$ Zoe Robinson, ${ }^{1}$ \\ Natalie Geoghegan, ${ }^{1}$ Katharine Jarrold, ${ }^{1}$ Thalia Afxentiou, ${ }^{1}$ Tim Reeves, ${ }^{2}$ Sergio Cunha, ${ }^{3}$ \\ Marialena Trivella, ${ }^{4}$ Vanessa Garcia-Larsen, ${ }^{2}$ Jo Leonardi-Bee ${ }^{5}$
}

'Section of Paediatrics, Imperial College London, London W2 1PG, UK

${ }^{2}$ Respiratory Epidemiology, Occupational Medicine and Public Health, National Heart and Lung Institute, Imperial College London, London SW3 6LR, UK

${ }^{3}$ Imperial Consultants, London SW7 2PG, UK

${ }^{4}$ Centre for Statistics in

Medicine, University of Oxford, Oxford OX3 7LD, UK

${ }^{5}$ Division of Epidemiology and Public Health, University of

Nottingham, Nottingham NG5 1PB, UK

Correspondence to: RBoyle r.boyle@nhs.net

Additional material is published online only. To view please visit the journal online.

Cite this as: BMJ 2016;352:i974 http://dx.doi.org/10.1136/bmj.i974

Accepted: 30 January 2016

\section{ABSTRACT}

OBJECTIVE

To determine whether feeding infants with hydrolysed formula reduces their risk of allergic or autoimmune disease.

DESIGN

Systematic review and meta-analysis, as part of a series of systematic reviews commissioned by the UK Food Standards Agency to inform guidelines on infant feeding. Two authors selected studies by consensus, independently extracted data, and assessed the quality of included studies using the Cochrane risk of bias tool.

\section{DATA SOURCES}

Medline, Embase, Web of Science, CENTRAL, and LILACS searched between January 1946 and April 2015.

ELIGIBILITY CRITERIA FOR SELECTING STUDIES Prospective intervention trials of hydrolysed cows' milk formula compared with another hydrolysed formula, human breast milk, or a standard cows' milk formula, which reported on allergic or autoimmune disease or allergic sensitisation.

\section{RESULTS}

37 eligible intervention trials of hydrolysed formula were identified, including over 19000 participants. There was evidence of conflict of interest and high or unclear risk of bias in most studies of allergic outcomes and evidence of publication bias for studies of eczema and wheeze. Overall there was no consistent evidence that partially or extensively hydrolysed formulas reduce risk of allergic or autoimmune outcomes in infants at high pre-existing risk of these outcomes. Odds ratios for eczema at age 0-4, compared with standard cows' milk formula, were 0.84 (95\% confidence interval 0.67 to $1.07 ;\left.\right|^{2}=30 \%$ ) for partially hydrolysed formula; 0.55 (0.28 to 1.09; $\left.\mathrm{I}^{2}=74 \%\right)$ for extensively hydrolysed casein based formula; and 1.12 ( 0.88 to $1.42 ; I^{2}=0 \%$ ) for extensively

\section{WHAT IS ALREADY KNOWN ON THIS TOPIC}

Breastfeeding is the optimum mode of nutrition for infants

Substitution with infant formula has been associated with allergic and autoimmune disease

International guidelines recommend use of a hydrolysed formula in place of standard infant formula for infants at risk of allergic disease to prevent eczema and allergy to cows' milk

\section{WHAT THIS STUDY ADDS}

There is no consistent evidence to support the use of hydrolysed formula for the prevention of allergic or autoimmune disease hydrolysed whey based formula. There was no evidence to support the health claim approved by the US Food and Drug Administration that a partially hydrolysed formula could reduce the risk of eczema nor the conclusion of the Cochrane review that hydrolysed formula could prevent allergy to cows' milk. CONCLUSION

These findings do not support current guidelines that recommend the use of hydrolysed formula to prevent allergic disease in high risk infants.

REVIEW REGISTRATION

PROSPERO CRD42013004252.

\section{Introduction}

Immune mediated health conditions such as allergic and autoimmune diseases seem to have increased in prevalence in many countries and are leading causes of chronic illness in young people. ${ }^{1}$ There is evidence that dietary exposures in infancy can influence the risk of these diseases, with a specific concern that early exposure to intact cows' milk protein in the form of infant formula could trigger the onset of allergic or autoimmune disease. ${ }^{2-4}$ Current infant feeding guidelines in North America, Australasia, and Europe recommend the use of hydrolysed formula in the first 4-6 months of life in place of a standard cows' milk formula for the primary prevention of allergic diseases in childhood..$^{5-7}$ This has also been supported by the US Food and Drug Administration (FDA) ${ }^{8}$ and by a Cochrane systematic review. ${ }^{910}$ To inform guidelines on infant feeding in the United Kingdom, we undertook a systematic review of hydrolysed formula for preventing allergic sensitisation, allergic disease, or autoimmune disease.

\section{Methods}

This review is reported in accordance with PRISMA guidance. The review is one of a series of systematic reviews commissioned by the UK Food Standards Agency to inform UK guidelines on infant feeding, under the title "review of scientific published literature on infant feeding and development of atopic and autoimmune disease." The protocols for the systematic reviews were registered with the International Prospective Register of Systematic Reviews (PROSPERO CRD42013003802 “milk feeding”; CRD42013004239 "timing of allergenic food introduction”; CRD42013004252 “maternal and infant diet”) on 5 August 2013, before titles were screened or studies selected from the search results. This review of hydrolysed formula is part of CRD42013004252 "maternal 
and infant diet." As part of this project we also searched for other systematic reviews covering the same topic published since 1 January 2011 with a revised AMSTAR score $\geq 32 .{ }^{11}$ No such reviews were identified for hydrolysed formula.

\section{Study interventions and comparators}

We included studies of any hydrolysed formula of cows' milk origin as the intervention of interest, compared with any non-hydrolysed cows' milk formula, human milk, or another type of hydrolysed cows' milk formula. Also included were studies in which hydrolysed formula was given as part of a multifaceted intervention, which we defined as an intervention with at least two other components in addition to the hydrolysed formula-for example, exclusion of allergenic food from the mother's diet, promotion of breastfeeding, delayed introduction of solid food, or measures to avoid exposure to house dust mite. We also included studies in which other interventions were applied to both intervention and control groups, such as exclusion of cows' milk from the mother's diet during lactation. Studies of hydrolysed formula of milk other than cows' milk, such as hydrolysed rice, goats' milk, or soya formula, were not included. We did not use a specific definition of partially or extensively hydrolysed formula but relied on the definition and/or trade name of formula as stated in the individual studies.

\section{Study designs and populations}

We included all intervention trials and searched for observational studies but did not extract data from these because of the evidence from intervention trials. Intervention trials were classified as randomised controlled trials, in which the method of treatment allocation was random; as quasi-randomised controlled trials, in which the method of treatment allocation was not totally random (but was thought unlikely to lead to imbalance between treatment groups in variables relevant to the outcome measures); and as controlled clinical trials, in which treatment allocation was non-random and likely to lead to imbalance between treatment groups. Controlled clinical trials were analysed separately from randomised controlled trials/quasi randomised controlled trials. We included studies of infant feeding between birth and 12 months of age and excluded studies in which infants or their mothers were defined by the presence of a pre-existing disease state, including very low birth weight or premature infants.

\section{Study outcomes}

Atopic and autoimmune outcomes were selected on the basis of their population prevalence in children and young adults in the UK and/or other affluent nations. We included diseases with a prevalence of at least one in 1000 children/adolescents or young adults (aged <40) but did not include rarer diseases. Atopic outcomes included were asthma (categorised as wheeze, recurrent wheeze, atopic wheeze, bronchial hyper-reactivity, forced vital capacity, peak expiratory flow rate, forced expiratory volume in one second), eczema, allergic rhinitis and/or conjunctivitis, food allergy, allergic sensitisation (that is, skin prick or specific immunoglobulin E (sIgE) assessment, or total IgE level). Autoimmune outcomes included were type 1 diabetes mellitus (defined serologically and/or clinically), coeliac disease (defined serologically and/or clinically), inflammatory bowel disease, autoimmune thyroid disease, juvenile rheumatoid arthritis, vitiligo, or psoriasis. For atopic outcomes, we grouped age at assessment as 0-4, 5-14, $15-24,25-44,45-64$, and $\geq 65$. Because of a paucity of outcome data in adults, we pooled age groups $\geq 15$. Autoimmune outcomes were not stratified for analyses by age at outcome assessment.

\section{Data sources}

The search strategies included both text terms and subject heading terms where appropriate and were initially developed for Medline and then adapted for other databases. We searched the Cochrane Library (2013, issue 7), Embase (1947 to July 2013), LILACS (1982 to July 2013), Medline (1946 to July 2013), and Web of Science (1970 to July 2013) on 25 July 2013 and reran the searches on 17 April 2015. We included all studies published up to that date and studies in progress or completed but unpublished identified through http://apps.who.int/ trialsearch/. Peer reviewed publications and abstract publications were included if they contained data that had not subsequently been published as a peer reviewed publication. We reviewed the bibliography of eligible studies for possible additional publications and included all eligible publications, regardless of the language. Authors of eligible or potentially eligible studies were not contacted for original data. We extensively piloted and refined the search strategies to optimise sensitivity, comparing search results with those of other published systematic reviews. Appendix 1 shows the Medline search strategies for the complete systematic review project.

\section{Study selection}

Seven trained researchers (RJB, VG-L, DI, NG, KJ, JC, $\mathrm{ZR})$ screened titles and abstracts in duplicate. Two researchers screened titles independently and met to agree on inclusion. Their screening was checked by a third member of the team, and uncertainties were brought to a full team meeting for discussion. This procedure took place between February and April 2014 and in April and May 2015 (RJB, TK, VG-L), with weekly team meetings to discuss uncertainties about study eligibility. The full text of all potentially eligible studies was reviewed.

\section{Assessment of risk of bias}

We assessed risk of bias in included intervention studies with a modified version of the Cochrane Collaboration risk of bias tool, which assessed sequence generation and allocation concealment (selection bias), blinding of outcome assessors and validity of the outcome assessment tool (assessment bias), and 
incomplete outcome data (attrition bias; considered high where $<70 \%$ of randomised participants had outcome data available). We modified the tool by including an assessment of conflict of interest, judged as low when there was no evidence of industry involvement in study design, analysis, interpretation, or publication and no evidence that study authors received remuneration from relevant industry partners for other activities.

\section{Data extraction}

DI, VG-L, RJB, and JL-B developed, piloted, and refined an Excel data extraction form. Two researchers (RJB, and TK or TA) extracted data in duplicate. Disagreements and uncertainties about data coding were discussed within the team with leads as follows: RJB (clinical queries), VG-L (dietetic queries), DI (analysis and coding queries), and JL-B (study design and statistics queries). For foreign language studies, VG-L extracted data with a native speaker of the relevant language (see acknowledgements section). We extracted all relevant data from included studies, including data that we could not meta-analyse. Data were extracted either with raw frequencies or crude or adjusted effect estimates. We undertook random effect meta-analysis. When this was not possible or not appropriate we summarised study results in a narrative table.

\section{Data selection for analysis}

We extracted outcome data that adhered to the intention to treat principle in preference to data based on per protocol analyses. When studies included multiple intervention groups, we performed pairwise comparisons in which we split the number of events and no events in the unexposed/control group to prevent double counting. When studies reported data at multiple time points within one of our predefined age groupings, we extracted the most complete dataset available, beyond the intervention period (that is, from age 1 onwards). This is the dataset with the largest denominator or when the denominator is identical for multiple time points then the largest numerator (number of events) is used. When studies reported multiple assessments of the same outcome at the same time point, we selected clinical assessments in preference to serological assessments and skin prick in preference to assessment of specific IgE of allergic sensitisation. The GINI study ${ }^{12-15}$ used generalised estimating equations (GEE) to generate odds ratios in some of their publications. This represents the most complete data available from the study, so we selected this in preference to other GINI study data analyses. Raw data or risk ratios were not given in the generalised estimating equations reports. So for analyses that include such data from the GINI study we calculated odds ratios in place of risk ratios to be able to include the most complete GINI study data available in meta-analysis. For some meta-analyses generalised estimating equation data from the GINI trial were either not reported or could not be used because of multiple intervention groups, and in these meta-analyses we used the most complete non-generalised estimating equation data available.

\section{Data synthesis}

We presented pooled results for binary outcomes from intervention studies as risk ratios calculated from the frequencies given in the study or as odds ratios when pooled data included those from a generalised estimating equation analysis and/or adjusted effect estimates. We pooled risk ratios using the Mantel-Haenszel method (with continuity correction of 0.5 in studies with zero cell frequencies) and odds ratios using the generic inverse variance method in the statistical programme R (R version 3.1.0, 2014, www.r-project.org). Pooled data for continuous outcomes measured with similar scales are presented as mean differences with 95\% confidence intervals. Heterogeneity was quantified with $\mathrm{I}^{2}$ and classified as low ( $\left.<25 \%\right)$, moderate (5-50\%), high (50-75\%), or extreme ( $>75 \%$ ). Data were not pooled when $I^{2} \geq 80 \%$. We undertook subgroup analyses for meta-analyses with more than five studies and assessed for publication bias (small study bias) using funnel plots and Egger's asymmetry test when there were 10 or more studies in a meta-analysis. We undertook subgroup analyses according to low versus unclear/high overall risk of bias; high versus normal/low risk of disease; quasi-randomised controlled trial versus randomised controlled trial; low versus unclear/high risk of conflict of interest; multifaceted versus not multifaceted intervention; and casein versus whey dominant hydrolysate.

\section{Patient involvement}

The findings of this study form part of the UK government's review of advice to the general public on infant feeding. No patients were involved in setting the research question or the outcome measures, nor were they involved in developing plans for design or implementation of the study. No patients were asked to advise on interpretation or writing up of results.

\section{Results}

Our original search identified 16289 original titles. Screening of titles, abstracts, and full text yielded 52 studies (37 intervention trials) of hydrolysed formula, including over 19000 participants. There were 28 randomised controlled trials, six quasi-randomised controlled trials, and three controlled clinical trials describing allergic or autoimmune outcomes. Appendix 2 shows full details of the search results (PRISMA flow chart), and characteristics of included studies are summarised in tables A and B in appendix 3. Twenty three studies used partially hydrolysed formula, and in at least 15 cases this was one specific formula (Nan HA/Good Start/Nidina HA/Beba HA, Nestlé, Vevey, Switzerland). Eighteen studies used extensively hydrolysed formula, and five studies (three for extensively, two for partially hydrolysed formula) used hydrolysed formula as part of a multifaceted intervention. In 30 of 37 studies infants were 

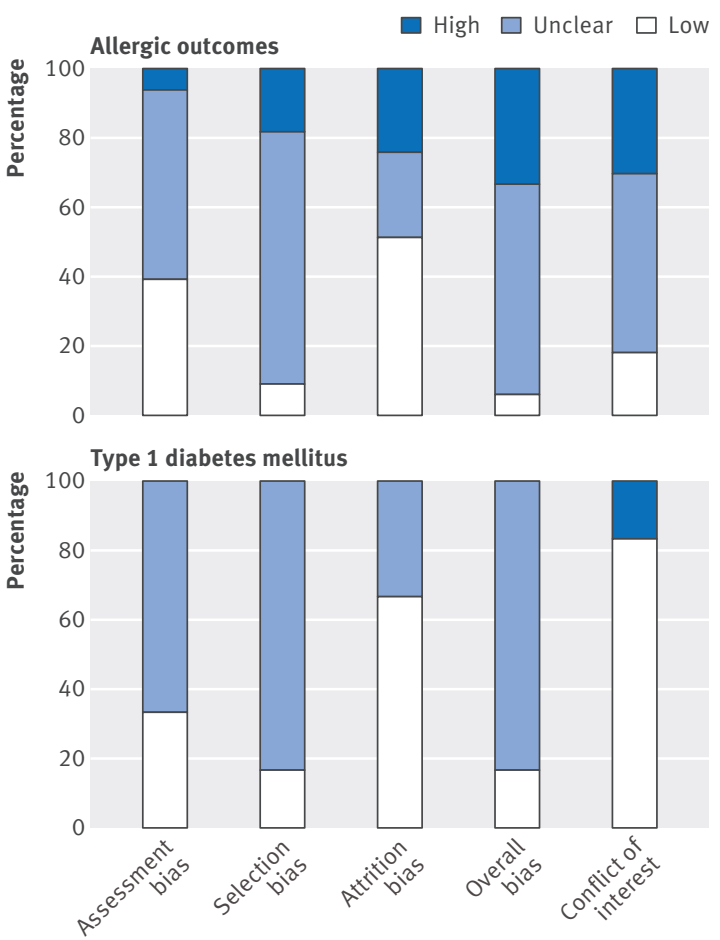

Fig 1 | Summary of risk of bias and conflict of interest in included studies reporting allergic outcomes and type 1 diabetes mellitus, showing proportion of studies with high, low, or unclear risk of bias in each domain

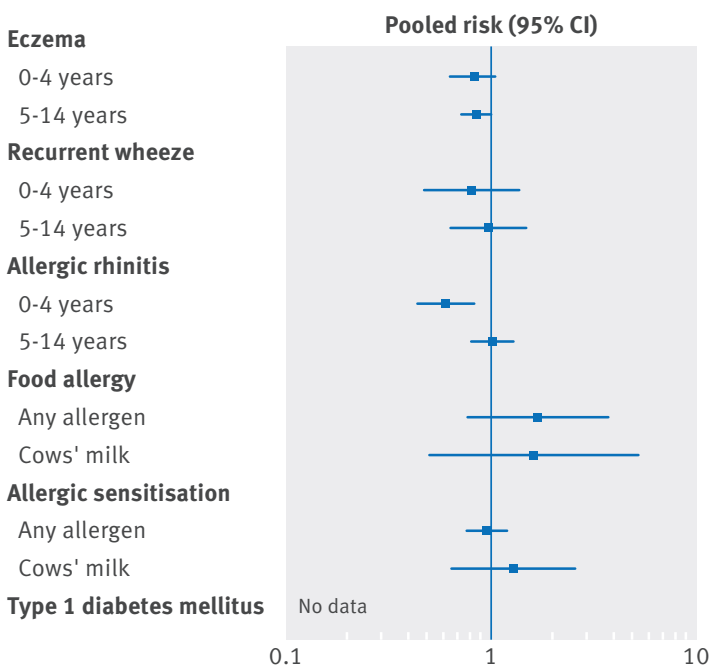

Fig 2 | Summary of treatment effects of hydrolysed formula on different outcome measures. Data shown are mean risk ratios (for allergic rhinitis at age 0-4; food allergy; allergic sensitisation; diabetes) or odds ratios (all other outcomes) with $95 \%$ confidence intervals for partially hydrolysed formula compared with standard cow's milk formula

at high risk of the relevant outcome(s) because of family history of disease in a first degree relative. Risk of bias and risk of conflict of interest were unclear or high in most studies for allergic outcomes (fig 1; table $\mathrm{C}$ in appendix 3), but low or unclear in most studies for autoimmune outcomes (fig 1 ; table D

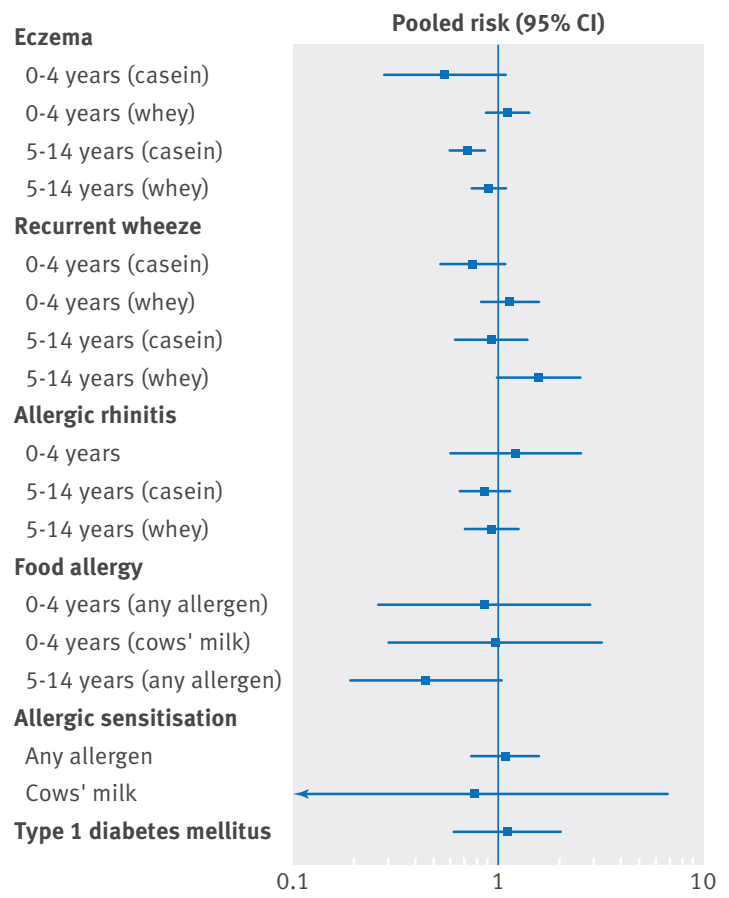

Fig 3 Summary of treatment effects of hydrolysed formula on different outcome measures. Data shown are mean risk ratios (for allergic rhinitis at age $0-4$; food allergy; allergic sensitisation; diabetes) or odds ratios (all other outcomes) with $95 \%$ confidence intervals for extensively hydrolysed formula compared with standard cows' milk formula

in appendix 3). Figure 2 (partially hydrolysed formula) and figure 3 (extensively hydrolysed formula) summarise the findings of the main meta-analyses. Table 1 shows the GRADE assessment and summary of findings for key comparisons. Figures 4-7 show meta-analyses reported in table 1 . The full report with a detailed description of all findings including meta-analyses and detailed methodology is available on the Food Standards Agency website (http://www.food.gov.uk/science/research/allergy-research/fs305005), together with an associated statement by the Committee on Toxicity (http://cot. food.gov.uk/cotstatements).

\section{Risk of eczema}

Twenty seven studies reported the effect of hydrolysed formula in infancy on risk of childhood eczema. The pooled data show no significant difference between extensively hydrolysed formula and standard cows' milk formula in risk of eczema at age 0-4 (odds ratio $0.84,95 \%$ confidence interval 0.67 to $1.07 ; \mathrm{I}^{2}=30 \%$ ) or age $5-14$ (0.86, 0.72 to $\left.1.02 ; \mathrm{I}^{2}=0 \%\right)$. Subgroup analysis suggested a significant difference in outcome according to study design or disease risk, with a more positive outcome in the single quasi-randomised controlled trial of normal risk infants. Analysis of data from randomised controlled trials for the most commonly studied partially hydrolysed formula (Nan HA/Good Start/Nidina HA/Beba HA, Nestlé, Vevey, Switzerland) showed no significant effect on risk of eczema at age 0-4 (0.94, 0.75 


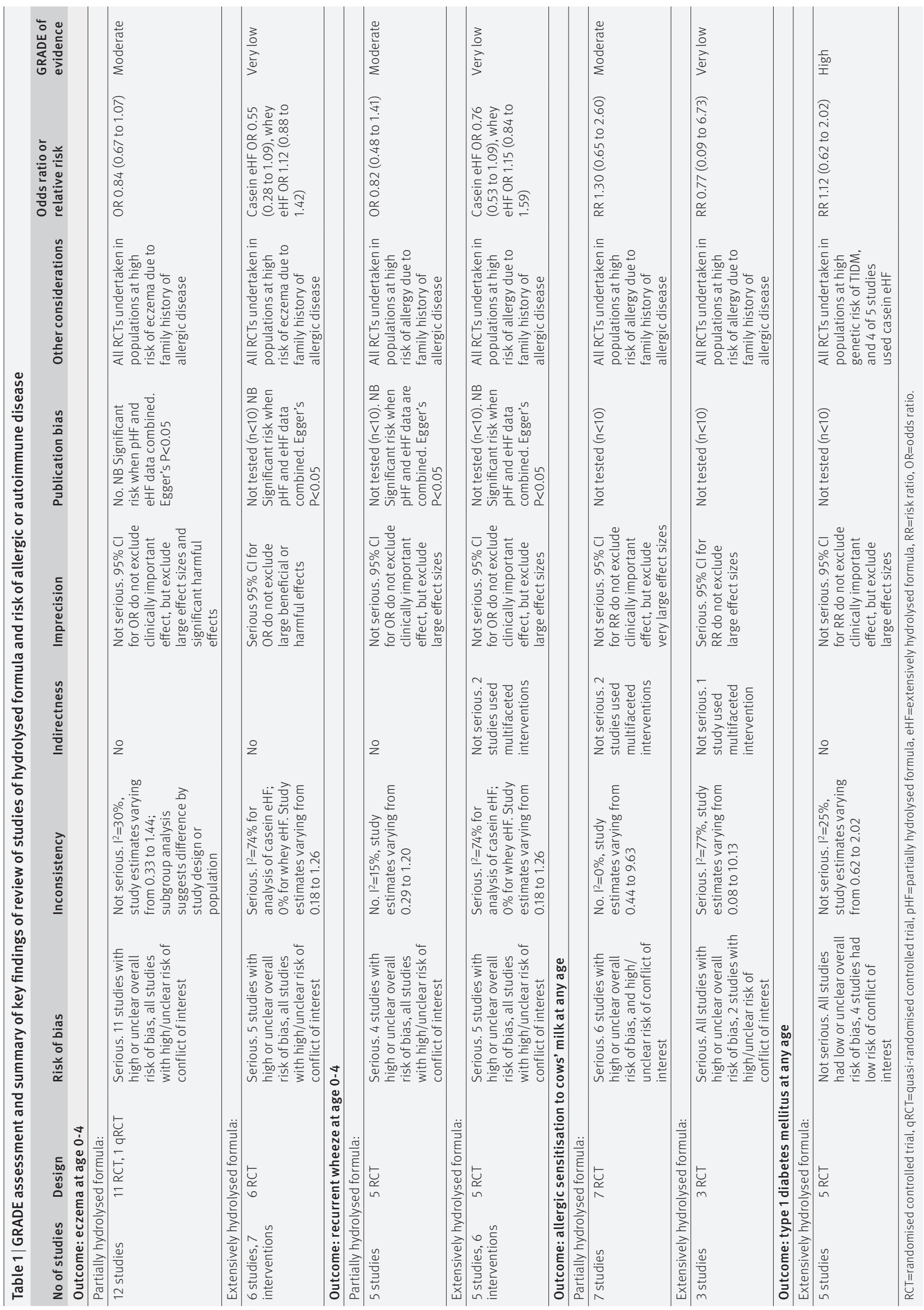




\section{Study}

Partially hydrolysed formula

Boyle 2014

Lowe 2011

Shao 2006

Von Berg 2003

Chan 2002

Exl 1998

Chirico 1997

Oldaeus 1997

Marini 1996

De Seta 1994

Vandenplas 1992

Tsai 1991

Random effects model

Test for heterogeneity: $P=0.15,\left.\right|^{2}=30 \%$

Extensively hydrolysed formula

Casein type formula

Von Berg 2003

Oldaeus 1997

Mallet 1992

Zeiger 1989

Random effects model

Test for heterogeneity: $\mathrm{P}=0.008, \mathrm{I}^{2}=74 \%$

Whey type formula

Schonberger 2005

Von Berg 2003

Lovegrove 1994

Random effects model

Test for heterogeneity: $\mathrm{P}=0.53, \mathrm{I}^{2}=0 \%$

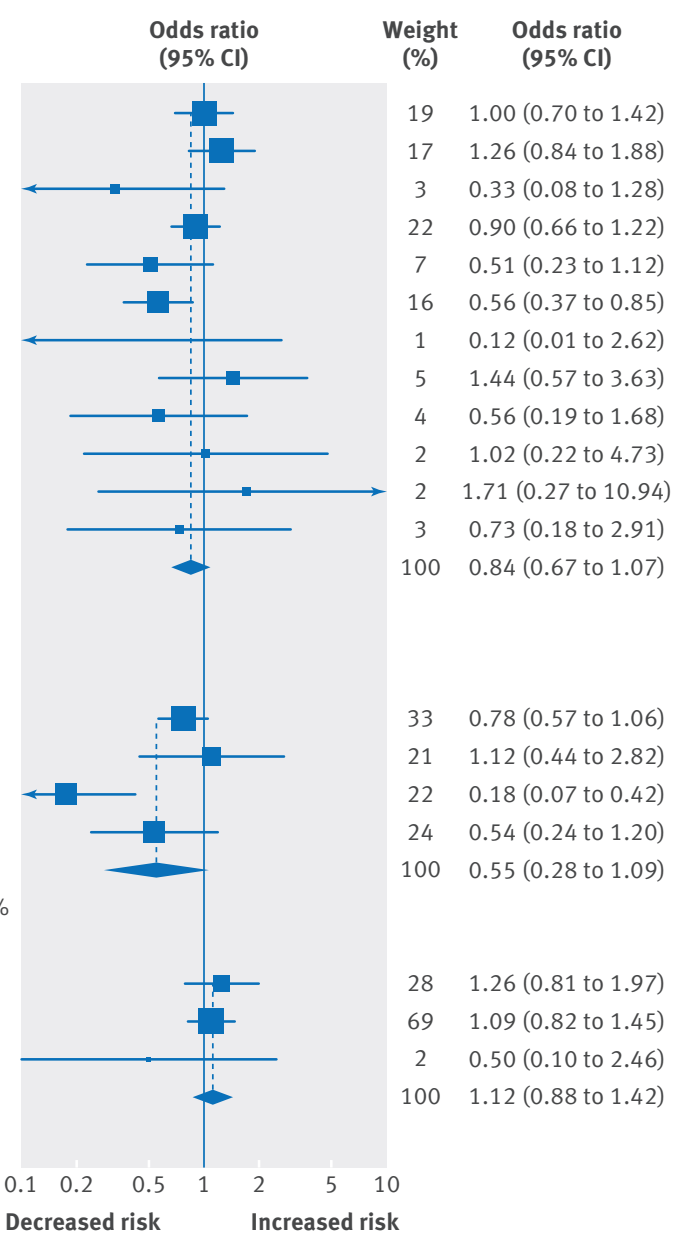

Fig 4 | Evidence from randomised or quasi-randomised controlled trials for partially hydrolysed formula and extensively hydrolysed formula compared with standard cows' milk formula for prevention of eczema in children aged $\leq 4$

\section{Study}

Partially hydrolysed formula

Von Berg 2006

Oldaeus 1997

Marini 1996

Vandenplas 1992

De Seta 1994

Random effects model

Test for heterogeneity: $P=0.32,1^{2}=15 \%$

Extensively hydrolysed formula

Casein type formula

Von Berg 2003

Oldaeus 1997

Mallet 1992

Zeiger 1989

Random effects model

Test for heterogeneity: $\mathrm{P}=0.91, \mathrm{I}^{2}=0 \%$

Whey type formula

Von Berg 2003

Schonberger 2005

Random effects mode

Test for heterogeneity: $\mathrm{P}=0.47, \mathrm{I}^{2}=0 \%$

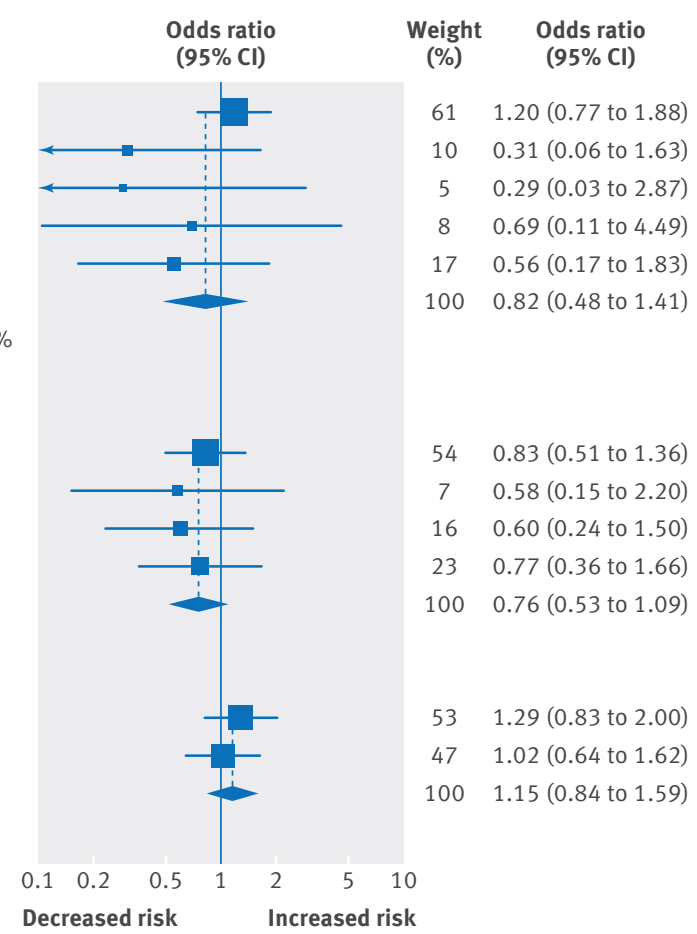

Fig 5 | Evidence from randomised controlled trials for partially hydrolysed formula and extensively hydrolysed formula compared with standard cow's milk formula, and recurrent wheezing at age $\leq 4$ 


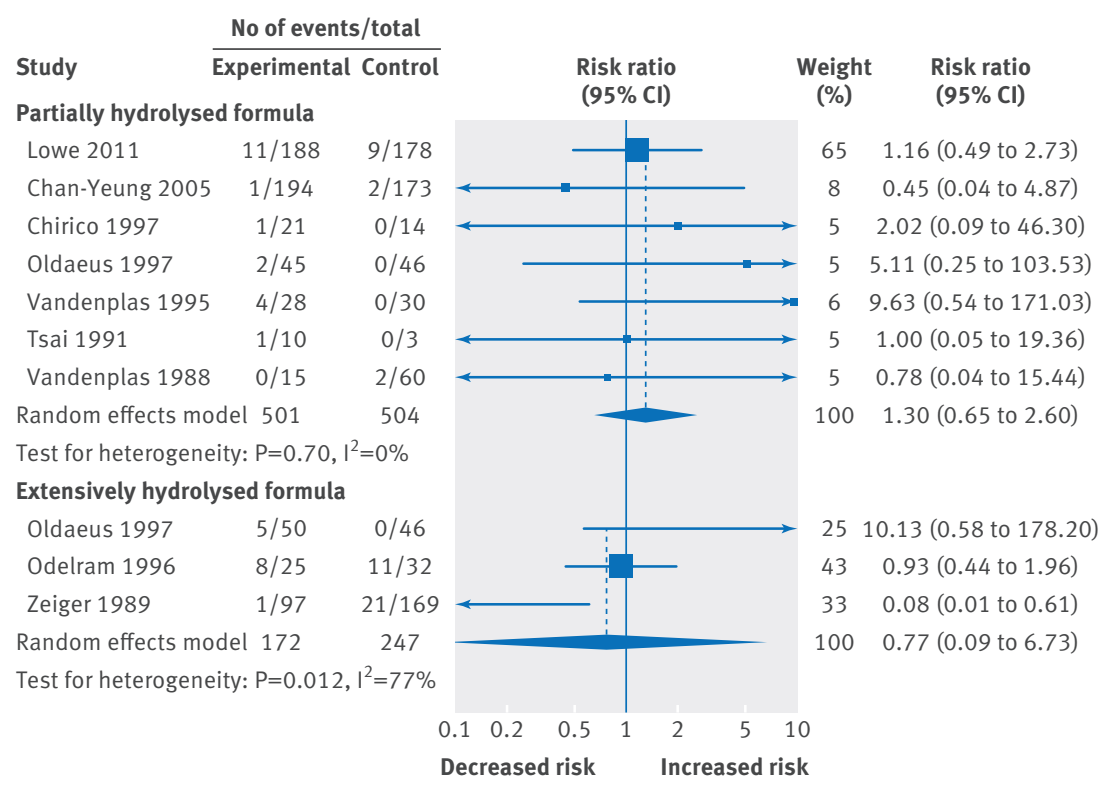

Fig 6 | Evidence from randomised controlled trials for partially hydrolysed formula and extensively hydrolysed formula compared with standard cow's milk formula, and allergic sensitisation to cows' milk

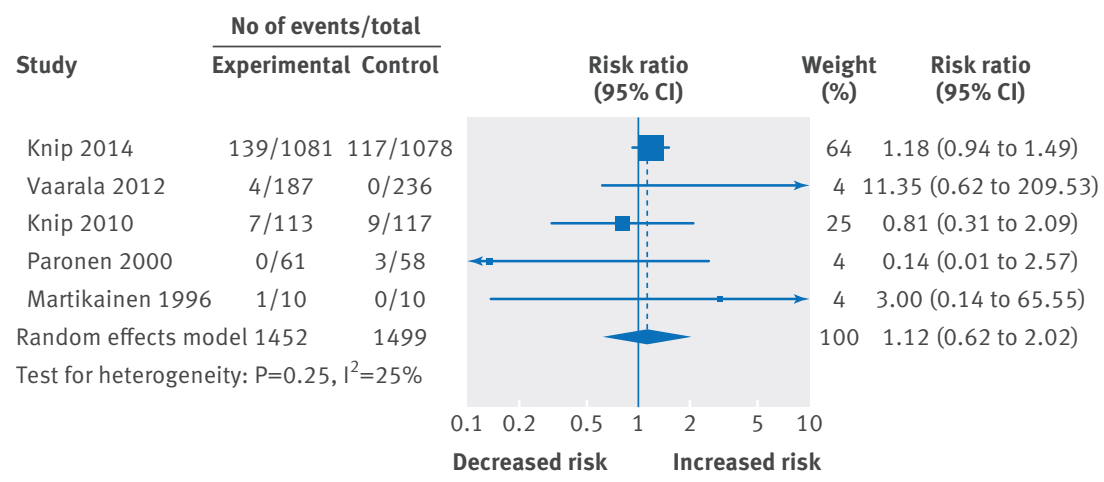

Fig 7 | Evidence from randomised controlled trials for extensively hydrolysed formula compared with standard cows' milk formula, and type 1 diabetes mellitus

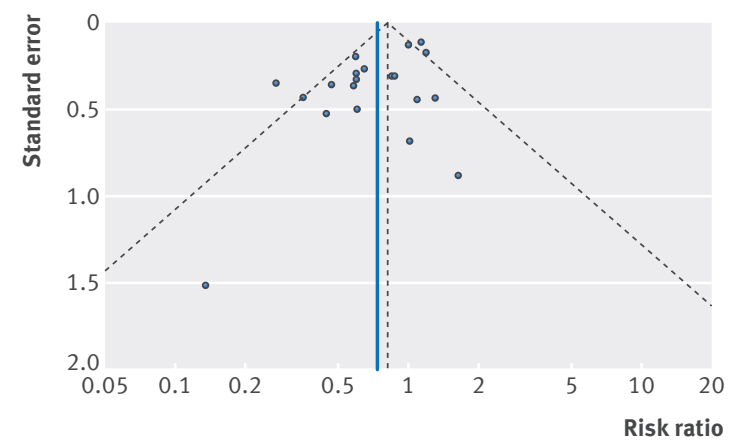

Fig 8 | Funnel plots for pooled analysis of "any hydrolysed formula" and risk of eczema showing evidence of publication bias/small study effects at age $\leq 4$ (Egger's test $\mathrm{P}=0.019$ )

to $\left.1.17 ; \mathrm{I}^{2}=0 \%\right)$. Though a meta-analysis of two studies on extensively hydrolysed formula found reduced odds of eczema at age 5-14 in children fed casein dominant formula during infancy $\left(0.71,0.59\right.$ to $\left.0.87 ; \mathrm{I}^{2}=0 \%\right)$, this effect was not seen for eczema at age 0-4 or for whey dominant formula at any age (0-4 or 5-14). Direct comparison of extensively versus partially hydrolysed formula, and casein versus whey dominant extensively hydrolysed formula, did not show a significant difference in risk of eczema at age $0-4$ or 5-14. When we combined data for partially and extensively hydrolysed formula as "any hydrolysed formula" there was evidence of reduced eczema at age 0-4 (but not at age 5-14). This analysis necessitated use of per protocol data from the GINI study, had high statistical heterogeneity, and showed evidence of publication bias (fig 8).

\section{Risk of wheeze}

Twenty one studies reported the effect of hydrolysed formula in infancy on risk of wheeze or recurrent wheeze. Analysis of the outcome "wheeze" was inconclusive, with meta-analyses of partially hydrolysed formula dominated by a multifaceted intervention study in which uptake of the intervention was low and a quasi-randomised controlled trial with high risk of bias and conflict of interest. Meta-analysis of extensively hydrolysed formula was not possible because of extreme heterogeneity. Analysis of the outcome "recurrent wheeze" showed no significant difference between partially hydrolysed formula and standard cows' milk formula for recurrent wheeze at age $0-4$ (odds ratio $0.82,95 \%$ confidence interval 0.48 to $1.41 ; \mathrm{I}^{2}=15 \%$ ) or age $5-14(0.99,0.65$ to $1.51 ; \mathrm{I}^{2}=43 \%$ ), and findings were also not significant for meta-analysis of casein or whey dominant extensively hydrolysed formula at ages 0-4 or 5-14. Direct comparison of extensively versus partially hydrolysed formula, and casein versus whey dominant extensively hydrolysed formula, did not show a significant difference in risk of recurrent wheeze at age $0-4$ or 5-14. When data for both types of formula were combined as "any hydrolysed formula" there was no evidence of reduced recurrent wheeze at age 0-4 or age 5-14-this analysis necessitated use of per protocol data for the GINI study, and showed evidence of publication bias (fig 9).

\section{Risk of allergic rhinitis}

Twelve studies reported the effect of hydrolysed formula on risk of allergic rhinitis. At age 0-4 partially

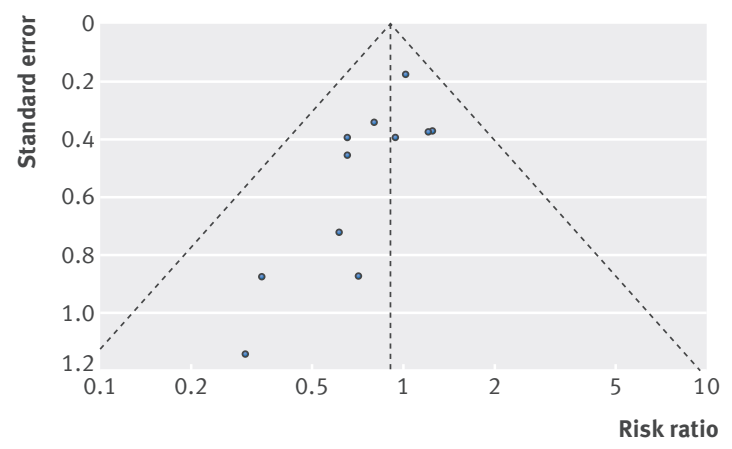

Fig 9 | Funnel plots for pooled analysis of "any hydrolysed formula" and recurrent wheeze showing evidence of publication bias/small study effects at age $\leq 4$ (Egger's test $\mathrm{P}=0.021$ ) 
hydrolysed formula (but not extensively hydrolysed formula) was associated with significantly reduced risk of allergic rhinitis, though this meta-analysis was dominated by a multifaceted intervention study in which uptake of the intervention was low. At age 5-14 neither partially (odds ratio 1.03, 95\% confidence interval 0.82 to $1.30 ; \mathrm{I}^{2}=0 \%$ ) nor extensively (casein $0.87,0.66$ to 1.15 ; $\mathrm{I}^{2}=0 \%$; whey $0.93,0.69$ to 1.26 ; single study) hydrolysed formula were associated with a significant reduction in allergic rhinitis. Direct comparison of the two formulas, and casein versus whey dominant extensively hydrolysed formula, did not show a significant difference in risk of allergic rhinitis at age $0-4$ or 5-14.

\section{Risk of food allergy or allergic sensitisation}

Thirteen and 19 studies reported the effect of hydrolysed formula on risk of food allergy and allergic sensitisation, respectively. There was no significant difference in risk of "any food allergy" with partially (risk ratio 1.73, 95\% confidence interval 0.79 to 3.80; $\left.\mathrm{I}^{2}=42 \%\right)$ or extensively (0.86, 0.26 to $\left.2.82 ; \mathrm{I}^{2}=42 \%\right)$ hydrolysed formula compared with standard formula at age 0-4, nor for extensively hydrolysed formula at age 5-14. We also found no difference in food allergy to cows' milk, egg, or (partially hydrolysed formula only) peanut. Direct comparison of the two formulas (egg allergy) and casein versus whey dominant extensively hydrolysed formula showed no significant difference in risk of food allergy. There was no significant difference in risk of allergic sensitisation to cows' milk with partially $\left(1.30,0.65\right.$ to $\left.2.60 ; \mathrm{I}^{2}=0 \%\right)$ or extensively $\left(0.77,0.09\right.$ to $\left.6.73 ; \mathrm{I}^{2}=77 \%\right)$ hydrolysed formula, and no significant difference between groups for risk of allergic sensitisation to "any allergen" or raised total IgE level.

\section{Risk of type 1 diabetes mellitus}

Six studies reported the effect of extensively hydrolysed formula compared with standard formula in infancy on risk of type 1 diabetes mellitus. There was no significant difference in risk with extensively hydrolysed formula (risk ratio 1.12, 95\% confidence interval 0.62 to 2.02; $\left.\mathrm{I}^{2}=25 \%\right)$. We did not identify any studies of partially hydrolysed formula and diabetes nor of hydrolysed formula and other autoimmune outcomes.

\section{Generalisability and study conduct}

In 18 of 33 (55\%) included studies reporting allergic outcomes, there were features suggesting issues with generalisability and possibly with study conduct. ${ }^{16}$ In seven studies there was either exclusive formula feeding from birth in all randomised participants ${ }^{17-22}$ or total cessation of breastfeeding by 14 days. ${ }^{23}$ In five studies, randomisation and allocation of formula milk occurred early: during pregnancy, ${ }^{24}$ at birth in all $^{2526}$ or some ${ }^{27}$ participants, or in the first four days after birth in most participants. ${ }^{28}$ In one study, infants were enrolled on day one, and over half were exclusively formula fed, ${ }^{29}$ and in another breast milk was purposefully withheld for the first three days of life in some infants. ${ }^{30}$ In two studies breastfeeding was not mentioned,,$^{31} 32$ and in another two studies ${ }^{33} 34$ it was unclear whether or not the study population was selected for a high rate of early formula feeding because of insufficient information in the report. The information presented in these studies suggests the possibility that more could have been done to promote the maintenance and revival of breastfeeding in the study populations, in accordance with the International Code of Marketing of Breast-milk Substitutes. ${ }^{16}$ In the 15 remaining studies of allergic disease $^{1235-48}$ and in all diabetes studies ${ }^{47}$ 49-52 there was no evidence of adverse breastfeeding outcomes in study participants.

\section{Discussion}

\section{Principal findings}

In this systematic review of hydrolysed infant formula for reducing risk of allergic or autoimmune disease we found no consistent evidence to support a protective role for partially or extensively hydrolysed formula. Our findings conflict with current international guidelines, in which hydrolysed formula is widely recommended for young formula fed infants with a family history of allergic disease. ${ }^{5-10}$ Such recommendations might therefore need revision.

\section{Comparison with other studies}

In 1936, Grulee and Sanford suggested that formula feeding could be associated with eczema, ${ }^{3}$ and more recent studies suggest that infant exposure to intact cows' milk might increase the risk of type 1 diabetes mellitus due to immunological cross reactivity between human and bovine insulin. ${ }^{4}$ Allergenic peptides in milk are 10-40 kDa in size, and bovine insulin is $5.8 \mathrm{kDa}$; partially and extensively hydrolysed formulas are intended to have no peptides $>5 \mathrm{kDa}$ and $\geq 3$ $\mathrm{kDa}$, respectively. ${ }^{53}$ By the avoidance of exposure to insulin or allergenic peptides in milk with partially or extensively hydrolysed formula, one might in theory prevent immunological sensitisation to insulin or milk and associated diseases such as type 1 diabetes, milk allergy, and eczema. We did not, however, find consistent evidence from intervention trials to support this.

The infant feeding guidelines that recommend hydrolysed formula for allergy prevention recognise that the evidence base for such a recommendation is weak and informed by analysis of a small number of trials with high risk of bias. In the current 2006 Cochrane review Osborn and colleagues concluded that there is limited evidence to support a role for hydrolysed formula in reducing allergy to cows' milk and no evidence for other specific allergic outcomes. ${ }^{910}$ This conclusion was based on a single study, ${ }^{21}$ in which the selected outcome is likely to be a poor measure of allergy to cows' milk as it was reported in over $40 \%$ of the control group, and such allergy has a prevalence of $2-7.5 \% .^{54}$ In our current meta-analysis of three studies reporting allergy to cows' milk, including data from the same study that 
defined allergy to cows' milk as challenge proved allergy with a positive allergy test result (3\% prevalence in control group), we found no evidence to support the conclusion of the Cochrane review.

International guidelines also support the use of hydrolysed formula for high risk infants to prevent eczema. ${ }^{5-7}$ We found no consistent evidence to support this. We did find that studies of lower quality design (controlled clinical trials and quasi-randomised controlled trials) had more positive outcomes than truly randomised controlled trials, and we found evidence of publication bias, suggesting that there could be small unpublished trials showing increased risk of eczema or recurrent wheeze associated with hydrolysed formula. The conclusions of an independent FDA review, which supported a limited health claim that a whey based partially hydrolysed formula (GoodStart, Nestlé, Vevey, Switzerland) could reduce the risk of eczema in high risk infants, also do not seem to be justified. ${ }^{8}$ That approval was based largely on review of the findings of the GINI study, in which per protocol analysis rather than intention to treat analysis was used to inform the FDA decision as the intention to treat analysis had not yet been published. ${ }^{8}$ The health claim was also approved before publication of one trial of the same formula with significantly negative results. ${ }^{42}$ Our own meta-analysis of published randomised controlled trials of the same partially hydrolysed formula does not support the FDA approved health claim that there is evidence for use of a whey based partially hydrolysed formula from birth to 4 months for reducing risk of eczema in healthy infants who are not exclusively breastfed and have a family history of allergy.

\section{Strengths and limitations of the study}

Many studies of allergic outcomes included in this review had unclear or high risk of bias and evidence of conflict of interest, often because of inadequate methods of randomisation and treatment allocation (selection bias) and support of the study or investigators from manufacturers of hydrolysed formula. In many cases study participants were infants with early full formula feeding, so our findings might not be applicable to populations with more typical feeding patterns.

Concerns have been raised about possible research fraud in this topic of investigation. We did not include the implicated studies, which evaluated a partially hydrolysed formula (Nan HA/Good Start/ Nidina HA/Beba HA, Nestlé, Vevey, Switzerland) for preventing allergic outcomes and were led by Chandra and colleagues. ${ }^{55-57}$ Data from the Chandra studies played an important role in a 2003 Cochrane review, which concluded that hydrolysed formula reduces allergic disease. ${ }^{58}$ Subsequent systematic reviews have excluded the Chandra data. The evidence of publication bias that we found, however, suggests that these systematic reviews might still overestimate the possibility that hydrolysed formula has beneficial effects.

\section{Conclusions and policy implications}

Our analyses suggest that current recommendations to use hydrolysed formula in place of standard cows' milk formula to prevent allergy in infants at high risk should be revised. We found no consistent evidence to support the current recommendations and found evidence of publication bias, methodological biases, and conflict of interest in those studies reporting allergic outcomes. We suggest that any future trials on hydrolysed formula should be prospectively registered, independently funded, and include adequate oversight to ensure that they do not negatively impact on breastfeeding in study participants.

We are grateful to the independent expert peer reviewers (Graham Devereux and Carina Venter) and to members of the UK Food Standards Agency, the Committee on Toxicity of Chemicals in Food, Consumer Products and the Environment (COT), and the UK Scientific Advisory Committee on Nutrition (SACN) for their comments on the study protocol and versions of the full report; Yujie Zhao, Szymon Mikolajewski, Andre Amaral, Mari Kihara, Christian Nielsen, Radoslav Latinovic, Stephanie MacNeill, Andreas Forsters, Daniel Munblit, Sze-Chin Tan, and Claudia Gore for their assistance with translation of foreign language reports; Jackie Cousins of the St Mary's library at Imperial College London for assistance with literature search training; Evangelia Andreou for assistance with creating tables and figures for the full report; and Dianne Campbell for commenting on a previous version of this manuscript.

Contributors: RJB, VG-L, and JL-B designed the study and wrote the protocol. RJB supervised data collection and synthesis and wrote the report and final draft of the manuscript. TR wrote the search strategy and undertook the literature search. TK, JC, ZR, NG, KJ, SC, and TA undertook title screening, data extraction, and data cleaning, supervised by RJB, VG-L, and DI. DI undertook all data analysis. MT and $J L-B$ advised on methods, statistical analyses, and interpretation of findings. VG-L and JL-B contributed equally to this work. All authors contributed to the final manuscript. RJB is guarantor

Funding: This work was funded by the Food Standards Agency and supported by a National Institute for Health Research Biomedical Research Centre. The Food Standards Agency commissioned this work, commissioned external peer review of the study protocol, statistical methods, and study report and approved the decision to submit this manuscript for publication. The National Institute for Health Research was not directly involved in the study design, implementation, analysis or the decision to submit for publication.

Competing interests: All authors have completed the ICMJE uniform disclosure form at http://www.icmje.org/coi_disclosure.pdf and declare: RJB, VG-L, JL-B and DI received support from the UK Food Standards Agency for the submitted work. No other support was received from any organisation for the submitted work. The authors have no financial relationships with any organisations that might have an interest in the submitted work in the previous three years. RJB was a co-investigator and author of one of the trials included in this systematic review. The authors report no other relationships or activities that could appear to have influenced the submitted work. Ethical approval: Not required.

Transparency declaration: RJB affirms that this manuscript is an honest, accurate, and transparent account of the study being reported; that no important aspects of the study have been omitted; and that any discrepancies from the study as planned and registered have been explained.

Data sharing: Dataset and statistical code are available from the Food Standards Agency at www.food.gov.uk

This is an Open Access article distributed in accordance with the Creative Commons Attribution Non Commercial (CC BY-NC 3.0) license, which permits others to distribute, remix, adapt, build upon this work non-commercially, and license their derivative works on different terms, provided the original work is properly cited and the use is non-commercial. See: http://creativecommons.org/licenses/ by-nc/3.0/

\footnotetext{
Prescott SL. Early-life environmental determinants of allergic diseases and the wider pandemic of inflammatory noncommunicable diseases. J Allergy Clin Immunol 2013;131:2330. doi:10.1016/j.jaci.2012.11.019.
} 
2 Du Toit G, Roberts G, Sayre PH, et al. LEAP Study Team. Randomized trial of peanut consumption in infants at risk for peanut allergy. $N$ Engl J Med 2015:372:803-13. doi:10.1056/NEJMoa1414850. .25705822.

3 Grulee CG, Sanford HN. The influence of breast and artificial feeding on infantile eczema. J Pediatr 1936;9:223-5.doi:10.1016/ S0022-3476(36)80058-4

4 Vaarala O, Knip M, Paronen J, et al. Cow's milk formula feeding induces primary immunization to insulin in infants at genetic risk for type 1 diabetes. Diabetes 1999;48:1389-94. http://onlinelibrary. wiley.com/o/cochrane/clcentral/articles/791/CN-00164791/frame. htmldoi:10.2337/diabetes.48.7.1389

5 ASCIA. Infant Feeding Advice. Secondary Infant Feeding Advice 2008 http://www.allergy.org.au/images/stories/hp/info/ASCIA_Infant_ Feeding_Advice_2010.pdf

6 Fleischer DM, Spergel JM, Assa'ad AH, Pongracic JA. Primary prevention of allergic disease through nutritional interventions. J Allergy Clin Immunol Pract 2013;1:29-36. doi:10.1016/j.jaip.2012.09.003.

7 Muraro A, Halken S, Arshad SH, et al. EAACI Food Allergy and Anaphylaxis Guidelines Group. EAACI food allergy and anaphylaxis guidelines. Primary prevention of food allergy. Allergy 2014;69:590601. doi:10.1111/all.12398

8 Chung CS, Yamini S, Trumbo PR. FDA's health claim review: wheyprotein partially hydrolyzed infant formula and atopic dermatitis. Pediatrics 2012;130:e408-14. doi:10.1542/peds.2012-0333.

9 Osborn D, Sinn J. Systematic review of formulas containing hydrolysed protein for prevention of allergy. Allergy 2013;68(supp 97):627. doi:10.1111/all.12252[published.

10 Osborn DA, Sinn JJ. Formulas containing hydrolysed protein for prevention of allergy and food intolerance in infants. Cochrane Database Syst Rev 2006;4:CD003664.

11 Kung J, Chiappelli F, Cajulis OO, et al. From Systematic Reviews to Clinical Recommendations for Evidence-Based Health Care: Validation of Revised Assessment of Multiple Systematic Reviews (R-AMSTAR) for Grading of Clinical Relevance. Open Dent J 2010;4:84-91. doi:10.2174/1874210601004020084[published.

12 Von Berg A, Koletzko S, Grübl A, et al. German Infant Nutritional Intervention Study Group. The effect of hydrolyzed cow's milk formula for allergy prevention in the first year of life: the German Infant Nutritional Intervention Study, a randomized double-blind trial. J Allergy Clin Immunol 2003;111:533-40. doi:10.1067/ mai.2003.101.

13 Von Berg A, Filipiak-Pittroff B, Krämer U, et al. GINIplus study group. Preventive effect of hydrolyzed infant formulas persists until age 6 years: long-term results from the German Infant Nutritional Intervention Study (GINI). J Allergy Clin Immunol 2008;121:1442-7. http://onlinelibrary.wiley.com/o/cochrane/clcentral/articles/168/ CN-00640168/frame.htmldoi:10.1016/j.jaci.2008.04.021.

14 Berg Av, Krämer U, Link E, et al. GINIplus study group. Impact of early feeding on childhood eczema: development after nutritional intervention compared with the natural course - the GINIplus study up to the age of 6 years. Clin Exp Allergy 2010;40:627-36. http:// onlinelibrary.wiley.com/o/cochrane/clcentral/articles/291/ CN-00750291/frame.html20082618.

15 Von Berg A, Filipiak-Pittroff B, Krämer U, et al. GINIplus study group. Allergies in high-risk schoolchildren after early intervention with cow's milk protein hydrolysates: 10-year results from the German Infant Nutritional Intervention (GINI) study. J Allergy Clin Immunol 2013:131:1565-73. doi:10.1016/j.jaci.2013.01.006.

16 WHO. International Code of Marketing of Breast-milk Substitutes. World Health Organization, 1981.

17 Chan YH, Shek LP, Aw M, Quak SH, Lee BW. Use of hypoallergenic formula in the prevention of atopic disease among Asian children.J Paediatr Child Health 2002;38:84-8. doi:10.1046/j.1440-1754.2002.00725.x.

18 Moran JR. Effects of prolonged exposure to partially hydrolyzed milk protein. J Pediatr 1992;121:S90-4. doi:10.1016/ S0022-3476(05)81414-3.

19 Tsai YT, Chou CC, Hsieh KH. The effect of hypoallergenic formula on the occurrence of allergic diseases in high risk infants. Zhonghua Min Guo Xiao Er Ke Yi Xue Hui Za Zhi 1991;32:137-44

20 Vandenplas Y, Deneyer M, Sacre L, Loeb H. Preliminary data on a field study with a new hypo-allergic formula. Eur J Pediatr 1988;148:274-7. doi:10.1007/BF00441420.

21 Vandenplas Y. Atopy at 3 years in high-risk infants fed whey hydrolysate or conventional formula. Lancet 1992;339:1118. doi:10.1016/0140-6736(92)90716-G.

22 Willems R, Duchateau J, Magrez P, Denis R, Casimir G. Influence of hypoallergenic milk formula on the incidence of early allergic manifestations in infants predisposed to atopic diseases. Ann Allergy 1993;71:147-50

23 Scalabrin DM, Johnston WH, Hoffman DR, P'Pool VL, Harris CL, Mitmesser SH. Growth and tolerance of healthy term infants receiving hydrolyzed infant formulas supplemented with Lactobacillus rhamnosus GG: randomized, double-blind, controlled trial. Clin Pediatr (Phila) 2009;48:734-44. doi:10.1177/0009922809332682.
24 Nentwich I, Michková E, Nevoral J, Urbanek R, Szépfalusi Z. Cow’s milk-specific cellular and humoral immune responses and atopy skin symptoms in infants from atopic families fed a partially (pHF) or extensively (eHF) hydrolyzed infant formula. Allergy 2001;56:114456. doi:10.1111/j.1398-9995.2001x.00926.x.

25 Chirico G, Gasparoni A, Ciardelli L, De Amici M, Colombo A, Rondini G. Immunogenicity and antigenicity of a partially hydrolyzed cow's milk infant formula. Allergy 1997;52:82-8. doi:10.1111/j.1398-9995.1997. tb02549.x.

26 Shao J, Sheng J, Dong W, Li YZ, Yu SC. [Effects of feeding intervention on development of eczema in atopy high-risk infants: an 18-month follow-up study]. Zhonghua Er Ke Za Zhi 2006;44:684-7.

27 Porch MC, Shahane AD, Leiva LE, et al. Influence of breast milk, soy or two hydrolyzed formulas on the development of allergic manifestations in infants at risk. Nutr Res 1998;18:141324doi:10.1016/S0271-5317(98)00119-5.

28 Saarinen KM, Juntunen-Backman K, Järvenpää AL, et al. Supplementary feeding in maternity hospitals and the risk of cow's milk allergy: A prospective study of 6209 infants. J Allergy Clin Immunol 1999:104:457-61. doi:10.1016/S0091-6749(99)70393-3.

29 Marini A, Agosti M, Motta G, Mosca F. Effects of a dietary and environmental prevention programme on the incidence of allergic symptoms in high atopic risk infants: three years' follow-up. Acta Paediatr Suppl 1996;414:1-21. doi:10.1111/j.1651-2227.1996. tb14267.x.

30 Juvonen P, Månsson M, Jakobsson I. Does early diet have an effect on subsequent macromolecular absorption and serum IgE?] Pediatr Gastroenterol Nutr 1994;18:344-9. doi:10.1097/00005176199404000-00016.

31 Akimoto K, Saito H, Akasawa A, likura Y. [Preventative effect of a whey hydrolyzed formula (Nestle, NAN H.A.) on the development of allergic symptoms in infants]. Arerugi 1997; 46:1044-51.

32 de Seta L, Siani P, Cirillo G, Di Gruttola M, Cimaduomo L, Coletta S. [The prevention of allergic diseases with a hypoallergenic formula: a follow-up at 24 months. The preliminary results]. Pediatr Med Chir 1994;16:251-4.

33 Dupont C, Basuyau J, Soulaine P, et al. Breast-milk, partially hydrolyzed formula, and extensively hydrolyzed formula: Immediate and long-term effects in infants at risk of allergy. Allergy 2009;64:63.

34 Mallet E, Henocq A. Long-term prevention of allergic diseases by using protein hydrolysate formula in at-risk infants. J Pediatr 1992;121:S95-100. doi:10.1016/S0022-3476(05)81415-5.

35 Chan-Yeung M, Manfreda J, Dimich-Ward H, Ferguson A, Watson W, Becker A. A randomized controlled study on the effectiveness of a multifaceted intervention program in the primary prevention of asthma in high-risk infants. Arch Pediatr Adolesc Med 2000;154:65763. doi:10.1001/archpedi.154.7.657.

36 Boyle RJ, Brown N, Chiang WC, et al. Partially hydrolysed prebiotic supplemented whey formula for the prevention of allergic manifestations in high risk infants: a multicentre double blind randomised controlled trial. Clin Transl Allergy 2015;5(Suppl 3):P30. doi:10.1186/2045-7022-5-S3-P30.

37 Exl BM, Deland U, Wall M, et al. Zug-Frauenfeld nutritional survey ('Zuff Study'): Allergen-reduced nutrition in a normal infant population and its health-related effects: Results at the age of six months. Nutr Res 1998;18:1443-62doi:10.1016/S0271-5317(98)00121-3.

38 Halken S, Høst A, Hansen LG, Osterballe O. Preventive effect of feeding high-risk infants a casein hydrolysate formula or an ultrafiltrated whey hydrolysate formula. A prospective, randomized, comparative clinical study. Pediatr Allergy Immunol 1993;4:173-81. doi:10.1111/j.1399-3038.1993.tb00088.x.

39 Halken S, Hansen KS, Jacobsen HP, et al. Comparison of a partially hydrolyzed infant formula with two extensively hydrolyzed formulas for allergy prevention: a prospective, randomized study. Pediatr Allergy Immunol 2000;11:149-61. doi:10.1034/j.1399-3038.2000.00081.x.

40 Han YS, Park HY, Ahn KM, Lee JS, Choi HM, Lee SI. Short-term effect of partially hydrolyzed formula on the prevention of development of atopic dermatitis in infants at high risk. J Korean Med Sci 2003;18:547-51. doi:10.3346/jkms.2003.18.4.547.

41 Lovegrove JA, Hampton SM, Morgan JB. The immunological and long-term atopic outcome of infants born to women following a milk-free diet during late pregnancy and lactation: a pilot study. BrJ Nutr 1994;71:223-38. doi:10.1079/BJN19940129.

42 Lowe Al, Hosking CS, Bennett CM, et al. Effect of a partially hydrolyzed whey infant formula at weaning on risk of allergic disease in high-risk children: a randomized controlled trial. J Allergy Clin Immunol 2011;128:360-365.e4. doi:10.1016/j.jaci.2010.05.006.

43 Odelram H, Vanto T, Jacobsen L, Kjellman NI. Whey hydrolysate compared with cow's milk-based formula for weaning at about 6 months of age in high allergy-risk infants: effects on atopic disease and sensitization. Allergy 1996;51:192-5. doi:10.1111/j.1398-9995.1996.tb00063.x

44 Schmitz J, Digeon B, Chastang C, et alEffects of brief early exposure to partially hydrolyzed and whole cow milk proteins. J Pediatr 1992;121(5 Pt 2):S85-9. 
45 Schönberger HJAM, Dompeling E, Knottnerus JA, et al. The PREVASC study: the clinical effect of a multifaceted educational intervention to prevent childhood asthma. Eur Respir / 2005:25:660-70. doi:10.1183/09031936.05.00067704

46 Oldaeus G, Anjou K, Björkstén B, Moran JR, Kjellman NI. Extensively and partially hydrolysed infant formulas for allergy prophylaxis. Arch Dis Child 1997;77:4-10. doi:10.1136/adc.77.1.4.

47 Martikainen A, Saukkonen T, Kulmala PK, et al. Disease-associated antibodies in offspring of mothers with IDDM. Diabetes 1996:45:1706-10. doi:10.2337/diab.45.12.1706.

48 Zeiger RS, Heller S, Mellon MH, et al. Effect of combined maternal and infant food-allergen avoidance on development of atopy in early infancy: a randomized study. J Allergy Clin Immunol 1989;84:72-89. doi:10.1016/0091-6749(89)90181-4.

49 Åkerblom HK, Virtanen SM, Ilonen J, et al. National TRIGR Study Groups. Dietary manipulation of beta cell autoimmunity in infants at increased risk of type 1 diabetes: a pilot study. Diabetologia 2005;48:829-37. doi:10.1007/s00125-005-1733-3.

50 Knip M, Åkerblom HK, Becker D, et al. TRIGR Study Group. Hydrolyzed infant formula and early $\beta$-cell autoimmunity: a randomized clinical trial. JAMA 2014;311:2279-87. doi:10.1001/jama.2014.5610.

51 Vaarala O, Ilonen J, Ruohtula T, et al. Removal of Bovine Insulin From Cow's Milk Formula and Early Initiation of Beta-Cell Autoimmunity in the FINDIA Pilot Study. Arch Pediatr Adolesc Med 2012;166:608-14. doi:10.1001/archpediatrics.2011.1559

52 Paronen J, Knip M, Savilahti E, et al. Finnish Trial to Reduce IDDM in the Genetically at Risk Study Group. Effect of cow's milk exposure and maternal type 1 diabetes on cellular and humoral immunization to dietary insulin in infants at genetic risk for type 1 diabetes. Diabetes 2000;49:1657-65. doi:10.2337/diabetes.49.10.1657.
53 Bjorksten B. Comment on Antigen-reduced infant formulas. Acta Paediatr 1993;82:660-2. doi:10.1111/j.1651-2227.1993.tb12785.x.

54 Ludman S, Shah N, Fox AT. Managing cows' milk allergy in children. BMJ 2013;347:f5424. doi:10.1136/bmj.f5424. .24041704.

55 Chandra RK. Five-year follow-up of high-risk infants with family history of allergy who were exclusively breast-fed or fed partial whey hydrolysate, soy, and conventional cow's milk formulas. J Pediatr Gastroenterol Nutr 1997;24:380-8. doi:10.1097/00005176-19970400000005.

56 Chandra RK, Hamed A. Cumulative incidence of atopic disorders in high risk infants fed whey hydrolysate, soy, and conventional cow milk formulas. Ann Allergy 1991;67:129-32.

57 Chandra RK, Puri S, Hamed A. Influence of maternal diet during lactation and use of formula feeds on development of atopic eczema in high risk infants [correction in BMJ 1989;299:896] [retraction in BM] 2015;351:h5682]. BMJ 1989;299:228-30. doi:10.1136/ bmi.299.6693.228.

58 Osborn DA, Sinn J. Formulas containing hydrolysed protein for prevention of allergy and food intolerance in infants. Cochrane Data Syst Rev 2003;4:CD003664.

Appendix 1: Search strategy

Appendix 2: PRISMA flowchart

Appendix 3: Supplementary tables A-D 